\title{
A case of vocal tic: an unusual presentation of neurobrucellosis
}

\author{
G.I. Bayhan, ${ }^{1}$ G. Tanır, ${ }^{1}$ U. Ertan ${ }^{2}$ and S. Bodur ${ }^{3}$
}

\section{Introduction}

Brucellosis is the most widespread zoonosis in the world. The disease is endemic in countries bordering the Mediterranean $[1-3]$. It is a multisystem disease that may present with a broad spectrum of clinical manifestations and complications. Neurologic manifestations of brucellosis are rare, occurring in 3\%-5\% of patients with systemic brucellosis [4]. Nervous system manifestations are very heterogeneous, and include clinical syndromes like meningitis, encephalitis, myelitis, radiculitis and the involvement of cranial or peripheral nerves [2,5].

In this report we describe a case which presented with unexplained chronic cough, refusal to eat and constipation. The observation of "dry cough with a bark-like vocalizations" and psychiatric consultation established the diagnosis of tic disorder. After the diagnosis and treatment of neurobrucellosis as an underlying condition, vocal tic of the patient completely resolved.

\section{Case report}

A 11-year-old boy presented with a 3-month history of cough, nervousness, generalized arthralgia and headache. He had lost his appetite and been refusing any food intake for 1 month. He had been suffering from constipation and he had defecated only 1 time since the previous month. He had lost an estimated 4 kg weight.
On admission, physical examination of the patient was completely normal, including neurological signs. Dry cough with bark-like vocalizations was observed during hospitalization. These vocalizations were unrelenting and were precipitated by the presence of people in his room. It was also observed that the patient could not eat anything, for this reason a feeding tube was employed.

Initial laboratory studies showed a white blood cell count of $3600 / \mathrm{mm}^{3}$ with 44\% neutrophils, 46\% lymphocytes; haemoglobin $15.2 \mathrm{~g} / \mathrm{dL}$; haematocrit $43.6 \%$ and platelets $261000 /$ $\mathrm{mm}^{3}$. The erythrocyte sedimentation rate was $4 \mathrm{~mm} / \mathrm{h}$ and $\mathrm{C}$-reactive protein $1.89 \mathrm{mg} / \mathrm{L}$ (normal < $2.9 \mathrm{mg} / \mathrm{L}$ ). Biochemical investigations were normal except for a high total protein level of $(8.5 \mathrm{~g} / \mathrm{dL}$ (normal range $6-8 \mathrm{~g} / \mathrm{dL}$ ), albumin $4.6 \mathrm{~g} / \mathrm{dL}$ normal range 3.1-4.8 $\mathrm{g} / \mathrm{dL})$.

Investigations were performed to explore the chronic cough, anorexia, vomiting and constipation. Chest Xray was normal. Tuberculin skin test was negative. Mycoplasma pneumoniae IgM and IgG were negative by enzyme immunoassay. Vocal cord laryngoscopy was performed under general anaesthesia and revealed no oedema, swelling or inflammation of the vocal cords. Airway anatomy and vocal cord motion was normal. Ultrasonographic examination of the abdomen showed a mesenteric lymphadenopathy with nonpathological dimension. The child was given a barium swallow examination for refusal to eat and vomiting. The findings were suggestive of partial web in the duodenum. For this reason gastrointestinal endoscopy was performed. There were no stricture, oesophagitis, gastro-oesophageal reflux and gastric ulcer. Colonoscopy was performed to rule out organic reasons of constipation. There were no stenoses, ulcerations, intestinal obstruction, malignancies, inflammatory bowel disease, or diverticular disease.

After these extensive investigations, a psychiatric consultation was done. Psychiatric evaluation revealed good general appearance and motor activity, stable affect and complete orientation. The patient was communicative but his speech had been frequently interrupted by a vocal tic. Thought content of the patient was generally about his cough. He was evaluated as having vocal tic disorder. Treatment with risperidone and fluoxetine was initiated.

The presence of psychiatric findings which were unresponsive to 2 -week anti-depressive treatment, the high serum protein level, and leukopenia suggested that the patient might have an organic disease with neuropsychiatric symptoms. The history of the patient was re-evaluated and revealed the consumption of unpasteurized cheese. Rose bengal test, brucella tube agglutination and brucella Coombs' test were all negative. Enzyme immunoassay was positive for Brucella IgM and IgG. The child was diagnosed as having brucellosis and it was thought that the psychiatric findings

${ }^{7}$ Department of Paediatric Infectious Diseases; ${ }^{2}$ Department of Paediatrics; ${ }^{3}$ Department of Child and Adolescent Psychiatry, Dr Sami Ulus Maternity and Children's Health and Diseases Training and Research Centre, Ankara, Turkey (Correspondence to G.I. Bayhan:gibayhan@gmail.com).

Received: 08/09/11; accepted: 12/12/11 
of the patient might be attributed to brucellosis.

To investigate the neurobrucellosis lumbar puncture was performed. The cerebrospinal fluid (CSF) did not contain white blood cells, protein level was $23 \mathrm{mg} / \mathrm{L}$ (normal range $15-45 \mathrm{mg} / \mathrm{dL}$ ) and glucose level was $66 \mathrm{mg} / \mathrm{L}$ (normal 40-70 mg/dL). Bacterial cultures of the CSF were negative. Magnetic resonance imaging of the brain revealed normal findings. Brainstem auditory evoked potential (BAEP) investigation of the patient was normal. For visual evoked potential (VEP), P100 latency was prolonged bilaterally.

Treatment with antibrucella drugs (oral rifampicin $20 \mathrm{mg} / \mathrm{kg}$ per day, doxycycline $200 \mathrm{mg}$ /day and intravenous gentamicin $7.5 \mathrm{mg} / \mathrm{kg}$ per day) was administered at the 14th day of hospitalization. The patient's condition gradually improved and the psychiatric symptoms resolved completely after 13 days of the antibrucella treatment so risperidone and fluoxetine were stopped.

The patient was discharged from hospital 4 weeks later and antibrucella therapy was completed to 6 months. He was symptom free and had gained $1 \mathrm{~kg}$ at the 6-month follow-up.

\section{Discussion}

Brucellosis is a multisystem disease that may present with various clinical manifestations and complications. Neurobrucellosis is one of the complications and is rare in children, being reported in only $0 \%$ to $3.8 \%$ of children with brucellosis [1,6-12]. Paediatric neurobrucellosis studies are, however, scarce in the English scientific literature $[12,13]$. Meningoencephalitis is the most common neurologic manifestation. Other reported clinical presentations of neurobrucellosis are meningovascular involvement, parenchymatous dysfunction, peripheral neuropathy/radiculopathy, sensorial and motor abnormalities, cranial nerve involvement, epilepsy, brain abscess, subarachnoid haemorrhage $[4,6,14]$. In our patient, who had completely normal neurological examination findings, meningoencephalitis, subarachnoid haemorrhage and brain parenchymal lesion were excluded by CSF and MRI investigations. Although headache, mental inattention, and depression are common complaints in patients with neurobrucellosis, presentation with isolated psychiatric symptoms is rare [15-17]. Our patient had psychiatric symptoms include refusal eating, chronic irritating cough with barking and nervousness. These findings were attributed to vocal tic, which caused social difficulties for the patient, especially in school, along with nervousness and depressive signs.

We think that the patient's tic severely affected his social and daily functioning; for this reason medical treatment was commenced. Transient tics usually begin between 3 and 10 years of age and wax and wane over a period of 4 weeks to 1 year. The Diagnostic and Statistical Manual of Mental Disorders (DSM-IV-TR) criteria state that they must persist for less than 6 months [18]. Although the patient's age and the duration of the tic were consistent with transient tic, in the end, the diagnosis of brucellosis discarded a diagnosis of transient tic. The etiology of tics in general remains unclear.

The reported psychiatric manifestations in neurobrucellosis in adult patients are depression, amnesia, psychosis, nervousness, irritability, agitation, nightmares, impaired cognitive function, loss of perception, amnesia, personality disorder, various degrees of behavioural abnormalities and euphoria $[2,6,15-17,19]$. In a study of 27 adult patients with brucellosis (14 with manifest neurological manifestation and 13 without apparent neurological manifestation), it was demonstrated that the patients with brucellosis (neurobrucellosis and patients without neurological manifestations) had highly significant impairment in some cognitive function measures and had higher scores on depressive symptoms compared with controls [15]. In another study, changes in mental and emotional status of the neurobrucellosis patients were investigated in adults. All of the neurobrucellosis cases were diagnosed with mild depression [16]. Depression was not detected in the brucellosis patients without neurological involvement. The mean Hamilton Depression Rating Scale test score among neurobrucellosis patients improved significantly with anti-brucella treatment without antidepressive and antipsychotic treatment while in the brucellosis patients without neurological involvement no significant improvements were observed with antibrucella treatment [16]. A prospective analysis of 73 patients with brucellosis identified 13 (17.8\%) neurobrucellosis cases, 10 with chronic and 3 with acute meningitis. Two patients with chronic meningitis presented only psychiatric disorders and headache [19].

Diagnosis of brucellosis is based upon serological tests and cultures. Blood culture is the gold standard in the diagnosis of brucellosis. Blood cultures are positive in $15 \%-90 \%$ of patients with brucellosis. In the absence of positive culture, the diagnosis can be made using serological testing with a variety of agglutination tests such as the rose bengal test, serum agglutination test, etc. The sensitivity of the serological tests ranges from $65 \%$ to $95 \%$, but their specificity is low because of the high prevalence of antibodies in the healthy population. The lack of seropositivity in patients with brucellosis may be attributed to the performance of tests early in the course of infection, decreased serum agglutination test titre in subacute or chronic cases, or the presence of blocking antibodies $[1,20,21]$. Enzyme-linked immunosorbent assay (EIA) is more sensitive than other serological tests, especially when the detection of specific IgM antibodies is complemented with the detection of 
specific IgG antibodies $[20,22]$. The specificity of EIA, however, seems to be lower than that of the agglutination tests. Serological testing with agglutination tests and EIA has been applied in the diagnosis of central nervous system brucellosis with varying success, and further research is aimed to improve the diagnosis of this condition [20]. In our patient, the lack of objective clinical findings and paucity of laboratory abnormalities initially suggested the diagnosis of depression, and treatment with anti-psychotic drugs was initiated. The final diagnosis of neurobrucellosis was by positive testing for brucella IgM, the history of raw milk ingestion and the rapid response to a specific treatment protocol.

We conclude that tic disorder during untreated neurobrucellosis in children is possible, and treatment of brucellosis may be associated with dramatic recovery of tic and comorbid conditions. Presentation with only psychiatric manifestations is extremely rare, especially in children, and clinicians should keep in mind that neurobrucellosis should be suspected in patients who experience unexplainable/unusual psychiatric problems, especially in areas endemic for brucellosis.

\section{References}

1. Tanir G, Tufekci SB, Tuygun N. Presentation, complications, and treatment outcome of brucellosis in Turkish children, Pediatrics International, 2009, 51:114-119.

2. Gul HC et al. Management of neurobrucellosis:an assessment of11 cases. InternalMedicine (Tokyo, Japan), 2008, 47:995-1001.

3. Buzgan $\mathrm{T}$ et al. Clinical manifestations and complications in 1028 cases of brucellosis: a retrospective evaluation and review of the literature. International Journal of Infectious Diseases, 2010, 14:469-478.

4. Yetkin MA et al. Evaluation of the clinical presentations in neurobrucellosis. International Journal of Infectious Diseases, 2006, 10:446-452.

5. Bucher A, Gaustad P, Pape E. Chronic neurobrucellosis due to Brucella melitensis. Scandinavian Journal of Infectious Diseases, 1990, 22:223-226.

6. Al Shaalan M et al. Brucellosis in children: clinical observations in 115 cases. International Journal of Infectious Diseases, 2002, 6:182-186

7. Tsolia M et al. Clinical features, complications and treatment outcome of childhood brucellosis in central Greece. Journal of Infection, 2002, 44(4):257-262.

8. Mantur BG et al. Childhood brucellosis-a microbiological, epidemiological and clinical study. Journal of Tropical Pediatrics, 2004, 50(3):153-157.

9. Caksen $\mathrm{H}$ et al. Childhood brucellosis is still a severe problem in the eastern region of Turkey. Tropical Doctor, 2002, 32(2):91-92.

10. Gür A et al. Complications of brucellosis in different age groups: a study of 283 cases in southeastern Anatolia of Turkey. Yonsei Medical Journal, 2003, 44(1):33-44.

11. Giannakopoulos I et al. Presentation of childhood brucellosis in Western Greece. Japanese Journal of Infectious Diseases, 2006, 59(3):160-163.
12. Lubani MM et al. Neurobrucellosis in children. Pediatric Infectious Disease Journal, 1989, 8(2):79-82.

13. Al-Eissa YA. Clinical and therapeutic features of childhood neurobrucellosis. Scandinavian Journal of Infectious Diseases, 1995, 27(4):339-343.

14. Bilen $\mathrm{S}$ et al. Four different clinical manifestations of neurobrucellosis (case reports). European Journal of Internal Medicine, 2008, 19:75-77.

15. Ghaydaa A et al. Neuropsychiatric evaluation of patients with brucellosis. Journal of Neurovirology, 2010, 16:48-55.

16. Eren $\mathrm{S}$ et al. Cognitive and emotional changes in neurobrucellosis. Journal of Infection, 2006, 53:184-189.

17. Gul HC, Erdem H, Bek S. Overview of neurobrucellosis: a pooled analysis of 187 cases. International Journal of Infectious Diseases, 2009, 13(6):339-343.

18. Diagnostic and statistical manual of mental disorders, 4th ed (text revision) (DSM-IV-TR). Arlington, Virginia, American Psychiatric Association, 2000

19. Bodur $\mathrm{H}$ et al. Neurobrucellosis in an endemic area of brucellosis. Scandinavian Journal of Infectious Diseases, 2003, 35:94-97.

20. Franco MP et al. Human brucellosis. Lancet Infectious Diseases, 2007, 7:775-786.

21. Alişkan H. Kültür ve serolojik yöntemlerin insan brusellozu tanisindaki değeri [The value of culture and serological methods in the diagnosis of human brucellosis]. Mikrobiyoloji Bulteni, 2008, 42(1):185-195.

22. Al Dahouk S et al. Laboratory-based diagnosis of brucellosis-a review of the literature. Part II: serological tests for brucellosis. Clinical Laboratory, 2003. 49(11-12):577-589. 